Article

\title{
Some Characterizations of Semi-Invariant Submanifolds of Golden Riemannian Manifolds
}

\author{
Mustafa Gök ${ }^{1, *(D)}$, Sadık Keleş ${ }^{2}$ (D) and Erol Kılıç ${ }^{2}$ (D) \\ 1 Department of Computer Technology, Sivas Cumhuriyet University, 58140 Sivas, Turkey \\ 2 Department of Mathematics, Inonü University, 44280 Malatya, Turkey; sadik.keles@inonu.edu.tr (S.K.); \\ erol.kilic@inonu.edu.tr (E.K.) \\ * Correspondence: mustafa.gok@email.com or mgok@cumhuriyet.edu.tr
}

Received: 30 September 2019; Accepted: 5 December 2019; Published: 9 December 2019

\begin{abstract}
In this paper, we study some characterizations for any submanifold of a golden Riemannian manifold to be semi-invariant in terms of canonical structures on the submanifold, induced by the golden structure of the ambient manifold. Besides, we determine forms of the distributions involved in the characterizations of a semi-invariant submanifold on both its tangent and normal bundles.
\end{abstract}

Keywords: golden structure; golden Riemannian manifold; semi-invariant submanifold

MSC: 53C15; 53C25; 53C40

\section{Introduction}

The notion of a CR submanifold in Kaehlerian manifolds has been first defined by A. Bejancu [1] as a generalization of both complex and totally real submanifolds. Later, this notion has been considered in Riemannian manifolds endowed with almost contact structures. In this sense, the concept of a semi-invariant submanifold in almost contact metric manifolds has been introduced by A. Bejancu and N. Papaghuic [2] as analogous to that of the CR submanifold, in fact, semi-invariant submanifolds in Riemannian manifolds correspond to CR submanifolds in complex manifolds. Since then, it has become a popular topic in differential geometry. This notion has been extended to other ambient manifolds. Therefore, semi-invariant submanifolds in different kind of ambient manifolds have been defined and studied by many geometers, such as Kenmotsu manifolds [3], locally Riemannian product manifolds [4], Sasakian space forms [5], cosymplectic manifolds [6,7], almost contact manifolds [8,9], nearly Sasakian manifolds [10], Lorentzian para-Sasakian manifolds [11], nearly trans-Sasakian manifolds [12], Lorentzian Sasakian manifolds [13].

A research regarding the differential geometry of golden structures on manifolds has been initiated by M. C. Crâşmăreanu and C. E. Hreţcanu in [14]. In [14-16], the concepts of a golden Riemannian structure and a golden Riemannian manifold have been introduced, in additon, some properties of golden Riemannian manifolds have been analyzed. After that, various type of submanifolds of golden Riemannian manifolds have been investigated such as, invariant submanifolds, anti-invariant submanifolds, slant submanifolds, semi-slant submanifolds, hemi-slant submanifolds in [15-19]. In [20], the notion of a semi-invariant submanifold in golden Riemannian manifolds has been defined by F. E. Erdoğan and C. Yıldırım, then the authors have made an examination of the distributions involved in its definiton.

The main aim of this paper is to investigate some characterizations for any submanifold of a golden Riemannian manifold to be semi-invariant on both its tangent and normal bundles by using canonical structures on the submanifold, induced by the golden structure of the ambient manifold. 
Moreover, we find forms of the distributions specifying the characterizations of a semi-invariant submanifold on both its tangent and normal bundles.

The paper has three sections and is organized as follows: Section 2 includes some basic facts on golden Riemannian manifolds and their submanifolds. Section 3 is concerned with an investigation of characterizations of any semi-invariant submanifold in a golden Riemannian manifold. We obtain some results on its canonical structures induced by the golden structure of the ambient manifold. We find three necessary and sufficient conditions for any submanifold of a golden Riemannian manifold to be semi-invariant. Also, we get equivalent expressions for each of the associated distributions on its tangent and normal bundles. Finally, an example is presented.

\section{Preliminaries}

In this section, we briefly review some general properties concerning golden Riemannian manifolds and their submanifolds.

A non-trivial $C^{\infty}$-tensor field $\bar{\Phi}$ of type $(1,1)$ on a $C^{\infty}$-differantiable real manifold $\bar{M}$ is called a golden structure if it satisfies the equation

$$
\bar{\Phi}^{2}=\bar{\Phi}+I,
$$

where $I$ is the identity operator on the Lie algebra $\Gamma(T \bar{M})$ of differentiable vector fields on $\bar{M}$ [14-16]. In fact, the golden structure is a special case of the polynomial structure. The polynomial structure $f$ of degree $n$ is a non-zero $C^{\infty}$-tensor field of type $(1,1)$ on a $C^{\infty}$-differantiable manifold $\bar{M}$ verifying the algebraic equation

$$
Q(x)=x^{n}+a_{n} x^{n-1}+\cdots+a_{2} x+a_{1} I=0,
$$

where $f^{n-1}(p), f^{n-2}(p), \ldots, f(p), I$ are linearly independent for every point $p \in \bar{M}$. Also, the monic polynomial $Q(x)$ is named the structure polynomial [21]. That is, the golden structure $\bar{\Phi}$ is a polynomial structure of degree 2 with the structure polynomial $Q(x)=x^{2}-x-1$. If $(\bar{M}, \bar{g})$ is a Riemannian manifold endowed with a golden structure $\bar{\Phi}$ such that $\bar{g}$ and $\bar{\Phi}$ satisfying the relation

$$
\bar{g}(\bar{\Phi} X, Y)=\bar{g}(X, \bar{\Phi} Y)
$$

for any vector fields $X, Y \in \Gamma(T \bar{M})$, then $(\bar{g}, \bar{\Phi})$ is named a golden Riemannian structure and $(\bar{M}, \bar{g}, \bar{\Phi})$ is called a golden Riemannian manifold [14-16].

Let $M$ be any isometrically immersed submanifold of a golden Riemannian manifold $(\bar{M}, \bar{g}, \bar{\Phi})$ and we denote by the same symbol $\bar{g}$ the Riemannian metric induced on $M$. We define four operators $T, N, t$ and $n$ as follows:

$$
\begin{aligned}
T X & =(\bar{\Phi} X)^{\top}, \\
N X & =(\bar{\Phi} X)^{\perp}, \\
t U & =(\bar{\Phi} U)^{\top}
\end{aligned}
$$

and

$$
n U=(\bar{\Phi} U)^{\perp}
$$

for any vector fields $X \in \Gamma(T M)$ and $U \in \Gamma\left(T M^{\perp}\right)$, where $(\bar{\Phi} X)^{\top},(\bar{\Phi} U)^{\top} \in \Gamma(T M)$ and $(\bar{\Phi} X)^{\perp},(\bar{\Phi} U)^{\perp} \in \Gamma\left(T M^{\perp}\right)$. Then for any vector field $X$ tangent to $M$, the vector field $\bar{\Phi} X$ is given by the form

$$
\bar{\Phi} X=T X+N X .
$$

Similarly, for any vector field $U$ normal to $M$, we have

$$
\bar{\Phi} U=t U+n U
$$


Also, it is obvious that the operators $T: \Gamma(T M) \rightarrow \Gamma(T M)$ and $n: \Gamma\left(T M^{\perp}\right) \rightarrow \Gamma\left(T M^{\perp}\right)$ are an endomorphism, and the operators $N: \Gamma(T M) \rightarrow \Gamma\left(T M^{\perp}\right)$ and $t: \Gamma\left(T M^{\perp}\right) \rightarrow \Gamma(T M)$ are a bundle-valued 1-form. In addition, the operators $T$ and $n$ are $\bar{g}$-symmetric. That is,

$$
\bar{g}(T X, Y)=\bar{g}(X, T Y)
$$

and

$$
\bar{g}(n U, V)=\bar{g}(U, n V)
$$

for any vector fields $X, Y \in \Gamma(T M)$ and $U, V \in \Gamma\left(T M^{\perp}\right)$ [17]. Taking account of the definition of the golden structure in (1), we obtain from (8) and (9) that the following relations:

$$
\begin{gathered}
T+I=T^{2}+t N, \\
N=N T+n N, \\
t=T t+t n
\end{gathered}
$$

and

$$
n+I=n^{2}+N t .
$$

\section{Characterizations of Semi-Invariant Submanifolds}

The purpose of this section is to give some characterizations of any semi-invariant submanifold of a golden Riemannian manifold and its associated distributions.

At first, we recall that the concept of a semi-invariant submanifold in golden Riemannian manifolds. Any isometrically immersed submanifold $M$ of a golden Riemannian manifold $(\bar{M}, \bar{g}, \bar{\Phi})$ is called a semi-invariant submanifold if there exist two orthogonal complementary distributions $D$ and $D^{\perp}$ on $M$ satisfying the following conditions:

(a) $\bar{\Phi}\left(D_{p}\right)=D_{p} \subseteq T_{p} M$ ，

(b) $\bar{\Phi}\left(D_{p}^{\perp}\right) \subseteq T_{p} M^{\perp}$

for each point $p \in M$, where $D$ and $D^{\perp}$ are said to be $\bar{\Phi}$-invariant distribution and $\bar{\Phi}$-anti-invariant distribution, respectively [20].

Proposition 1. Let $M$ be any semi-invariant submanifold of a golden Riemannian manifold $(\bar{M}, \bar{g}, \bar{\Phi})$. Then we have the following expressions:

$$
\begin{aligned}
T D^{\perp} & =\{0\}, \\
N D^{\perp} & =\bar{\Phi} D^{\perp}, \\
N D & =\{0\}
\end{aligned}
$$

and

$$
T D=D .
$$

Proof. Since the distribution $D^{\perp}$ is $\bar{\Phi}$-anti-invariant, we have $\bar{\Phi}\left(D^{\perp}\right) \subseteq T M^{\perp}$, which proves (16). The proof of (17) is obvious from (8) and (16). As the distribution $D$ is $\bar{\Phi}$-invariant, it follows from (8) that (18) holds. By means of (8) and (10), we obtain

$$
\bar{g}(T X, Y)=\bar{g}(X, T Y)=\bar{g}(X, \bar{\Phi} Y)=0,
$$


for any vector fields $X \in \Gamma(D)$ and $Y \in \Gamma\left(D^{\perp}\right)$. Hence, (20) implies that $T D$ is perpendicular to $D^{\perp}$. At the same time, because of the fact that $T D \subseteq T M$, we get

$$
T D \subseteq D
$$

Let $X$ be in $\Gamma(D)$. Taking into account (18) in (9), we derive from (12) that

$$
D \subseteq T D
$$

Thus, we infer from (21) and (22) that (19) is correct. Consequently, the proof has been shown.

Theorem 1. Let $M$ be any submanifold of a golden Riemannian manifold $(\bar{M}, \bar{g}, \bar{\Phi})$. Then a necessary and sufficient condition for the submanifold $M$ to be a semi-invariant is that

$$
N T=0 .
$$

Proof. We assume that $M$ is a semi-invariant submanifold of the golden Riemannian manifold $(\bar{M}, \bar{g}, \bar{\Phi})$. Then the tangent bundle $T M$ has the decomposition $T M=D \oplus D^{\perp}$, where $D$ is $\bar{\Phi}$-invariant distribution and $D^{\perp}$ is $\bar{\Phi}$-anti invariant distribution. We denote by $r$ and $s$ the projection operators of the tangent bundle $T M$ onto the distributions $D$ and $D^{\perp}$, respectively. In this case, we have

$$
r+s=I, r^{2}=r, s^{2}=s \text { and } r s=s r=0 .
$$

Hence, for every vector field $X \in \Gamma(T M)$, there exists the decomposition

$$
X=r X+s X .
$$

From (24), $\bar{\Phi} X$ can be written in the form

$$
\bar{\Phi} X=\bar{\Phi} r X+\bar{\Phi} s X
$$

for any vector field $X \in \Gamma(T M)$. Then in view of (8), we get

$$
T X+N X=\operatorname{Tr} X+N r X+T s X+N s X
$$

for any vector field $X \in \Gamma(T M)$. On the other hand, we infer from (16) and (18) that

$$
T s=0 \text { and } N r=0 .
$$

Thus, comparing the tangential and normal parts of both sides of (25), we obtain

$$
T=\operatorname{Tr} \text { and } N=N s .
$$

Hence, we deduce from (13) and (18) that

$$
N T=0 .
$$

Conversely, let us suppose that $M$ is any submanifold of the golden Riemannian manifold $(\bar{M}, \bar{g}, \bar{\Phi})$ and $N T=0$. Applying the endomorphism $T$ from the right hand side to (12), we get

$$
T^{3}=T^{2}+T
$$


We define two operators as follows:

$$
r=T^{2}-T \text { and } s=-T^{2}+T+I .
$$

Then the operators $r$ and $s$ verify that

$$
r+s=I, r^{2}=r, s^{2}=s \text { and } r s=s r=0 .
$$

That is, $r$ and $s$ are orthogonal complementary projection operators. Hence, there are two orthogonal complementary distributions $D$ and $D^{\perp}$ corresponding to the projection operators $r$ and $s$, respectively. Taking into account the assumption that $N T=0$, we derive from (27) and (28) that

$$
\operatorname{Tr}=T, T s=0, s \operatorname{Tr}=s T=0 \text { and } N r=0,
$$

which imply that the distribution $D$ is $\bar{\Phi}$-invariant and the distribution $D^{\perp}$ is $\bar{\Phi}$-anti-invariant.

Consequently, we have two orthogonal complementary distributions $\bar{\Phi}$-invariant $D$ and $\bar{\Phi}$-anti-invariant $D^{\perp}$ on the submanifold $M$. In other words, $M$ is a semi-invariant submanifold.

Proposition 2. Let $M$ be any semi-invariant submanifold of a golden Riemannian manifold $(\bar{M}, \bar{g}, \bar{\Phi})$. Then we have the following expressions:

$$
\operatorname{ker} N=\operatorname{ker}\left(T^{2}-T-I\right)=\operatorname{ker} t N
$$

and

$$
\operatorname{ker} T=\operatorname{ker}\left(T^{2}-T\right)=\operatorname{ker}(t N-I) .
$$

Proof. Let $X$ be in $\Gamma(\operatorname{ker} N)$. Then it results from (12) that

$$
\operatorname{ker} N \subseteq \operatorname{ker}\left(T^{2}-T-I\right) .
$$

Conversely, if $X$ belongs to $\Gamma\left(\operatorname{ker}\left(T^{2}-T-I\right)\right)$, then we get from (12) that

$$
t N X=0 .
$$

On the other hand, using (3) and (8), we find

$$
\|N X\|^{2}=\bar{g}(X, t N X) .
$$

Hence, by means of (32) and (33), we obtain $N X=0$, from which

$$
\operatorname{ker}\left(T^{2}-T-I\right) \subseteq \operatorname{ker} N .
$$

Therefore, it follows from (31) and (34) that

$$
\operatorname{ker}\left(T^{2}-T-I\right)=\operatorname{ker} N .
$$

Besides, (12) states that

$$
\operatorname{ker}\left(T^{2}-T-I\right)=\operatorname{ker}(t N) .
$$

Consequently, it is seen immediately from (35) and (36) that (29) is true. Let $X \in \Gamma(\operatorname{ker} T)$. Then it is trivial that

$$
\operatorname{ker} T \subseteq \operatorname{ker}\left(T^{2}-T\right) .
$$


Conversely, if $X$ pertains to $\Gamma\left(\operatorname{ker}\left(T^{2}-T\right)\right)$, then we have

$$
T^{2} X=T X .
$$

Also, (12) shows that

$$
t N X=X
$$

Hence, using (3), (8), (9), (10), (38) and (39), we can easily find

$$
\|T X\|^{2}=\bar{g}(N T X, N X) .
$$

Then in view of (23), which is a necessary and sufficient condition for the submanifold $M$ to be semi-invariant, we obtain

$$
T X=0,
$$

from which

$$
\operatorname{ker}\left(T^{2}-T\right) \subseteq \operatorname{ker} T .
$$

Thus, by means of (37) and (41), we get

$$
\operatorname{ker}\left(T^{2}-T\right)=\operatorname{ker} T \text {. }
$$

On the other hand, it is obvious from (12) that

$$
\operatorname{ker} T=\operatorname{ker}(t N-I) \text {. }
$$

As a consequence of (42) and (43), we have (30). Therefore, the proof has been completed.

Proposition 3. Let $M$ be any semi-invariant submanifold of a golden Riemannian manifold $(\bar{M}, \bar{g}, \bar{\Phi})$. Then we have the following relations:

$$
D=\operatorname{ker} N=\operatorname{ker}\left(T^{2}-T-I\right)=\operatorname{ker}(t N)
$$

and

$$
D^{\perp}=\operatorname{ker} T=\operatorname{ker}\left(T^{2}-T\right)=\operatorname{ker}(t N-I) .
$$

Proof. The proof is a direct consequence of Proposition 1 and Proposition 2.

Let $M$ be any semi-invariant submanifold of a golden Riemannian manifold $(\bar{M}, \bar{g}, \bar{\Phi})$. We put

$$
\mathfrak{D}^{\perp}=\bar{\Phi} D^{\perp} .
$$

If we denote by $\mathfrak{D}$ the orthogonal complementary subbundle of $\mathfrak{D}^{\perp}$ in $T M^{\perp}$, then we have

$$
T M^{\perp}=\mathfrak{D} \oplus \mathfrak{D}^{\perp} .
$$

On the other hand, we consider a tensor field $\bar{\Psi}$ of type $(1,1)$ on the golden Riemannian manifold $(\bar{M}, \bar{g}, \bar{\Phi})$ defined by the rule

$$
\bar{\Psi}=I-\bar{\Phi} .
$$

In this case, $\bar{\Psi}$ is a golden structure [14]. Besides, the Riemannian metric $\bar{g}$ is $\bar{\Psi}$-compatible, that is, $\bar{g}$ and $\bar{\Psi}$ verifying the relation

$$
\bar{g}(\bar{\Psi} X, Y)=\bar{g}(X, \bar{\Psi} Y)
$$

for any vector fields $X, Y \in \Gamma(T \bar{M})$. 
Proposition 4. Let $M$ be any semi-invariant submanifold of a golden Riemannian manifold $(\bar{M}, \bar{g}, \bar{\Phi})$. Then we have the following expressions:

(a) $\mathfrak{D}$ is a $\bar{\Psi}$-invariant distribution,

(b) $\mathfrak{D}^{\perp}$ is a $\bar{\Psi}$-anti-invariant distribution.

Proof. Let $U$ be in $\Gamma(\mathfrak{D})$. Then we infer from (8) and (48) that

$$
\bar{g}(\bar{\Psi} U, X)=-\bar{g}(U, N X)
$$

for any vector field $X \in \Gamma(T M)$. Taking into account (17) and (18), it seems from (49) that

$$
\bar{g}(\bar{\Psi} U, X)=0,
$$

which implies

$$
\bar{\Psi} U \in \Gamma\left(T M^{\perp}\right) .
$$

Now, we assume that $V \in \Gamma\left(\mathfrak{D}^{\perp}\right)$. Then from (17), there exists a vector field $Y \in \Gamma\left(D^{\perp}\right)$ such that $V=\bar{\Phi} Y$. Thus, we have

$$
\bar{\Psi} V=-Y \in \Gamma\left(D^{\perp}\right) .
$$

Using (48) and (51), we find

$$
\bar{g}(\bar{\Psi} U, V)=\bar{g}(U, \bar{\Psi} V)=0,
$$

from which

$$
\bar{\Psi} U \in \Gamma(\mathfrak{D}) .
$$

It follows from (50) and (52) that $\mathfrak{D}$ is a $\bar{\Psi}$-invariant distribution. That is, we obtain (a). We recall that $\mathfrak{D}^{\perp}=\bar{\Phi} D^{\perp} \subseteq T M^{\perp}$. If $U \in \Gamma\left(\mathfrak{D}^{\perp}\right)$, then there is a vector field $Z$ in $\Gamma\left(D^{\perp}\right)$ such that $U=\bar{\Phi} Z$. Thus, we conclude

$$
\bar{\Psi} U=-Z \in \Gamma\left(D^{\perp}\right) \subseteq \Gamma(T M),
$$

which shows that $\mathfrak{D}^{\perp}$ is a $\bar{\Psi}$-anti-invariant distribution. In other words, we get (b).

Proposition 5. Let $M$ be any semi-invariant submanifold of a golden Riemannian manifold $(\bar{M}, \bar{g}, \bar{\Phi})$. Then we have the following relations:

$$
\begin{gathered}
t \mathfrak{D}=\{0\}, \\
n \mathfrak{D}=(I-n) \mathfrak{D}=\mathfrak{D} \text { or } \bar{\Phi} \mathfrak{D}=\mathfrak{D}, \\
(I-n) \mathfrak{D}^{\perp}=\{0\}
\end{gathered}
$$

and

$$
t \mathfrak{D}^{\perp}=D^{\perp} \text { or } \bar{\Psi} \mathfrak{D}^{\perp}=t \mathfrak{D}^{\perp}=D^{\perp} .
$$

Proof. Since the distribution $\mathfrak{D}$ is $\bar{\Psi}$-invariant, we have $\bar{\Psi} \mathfrak{D} \subseteq T M^{\perp}$, which implies from (9) that (53) holds. Taking account of that $\mathfrak{D}^{\perp}$ is $\bar{\Psi}$-anti-invariant distribution, we obtain from (9) and (48) that for any vector fields $U \in \Gamma(\mathfrak{D})$ and $V \in \Gamma\left(\mathfrak{D}^{\perp}\right)$,

$$
\bar{g}(n U, V)=-\bar{g}(U, \bar{\Psi} V)=0,
$$

which states

$$
n \mathfrak{D} \perp \mathfrak{D}^{\perp} .
$$


Also, by reason of the fact that $n \mathfrak{D} \subseteq T M^{\perp}$, it seems that

$$
n \mathfrak{D} \subseteq \mathfrak{D} .
$$

On the other hand, by means of (15), we derive from (53) and (59) that

$$
\mathfrak{D} \subseteq n \mathfrak{D}
$$

Hence, we get from (59) and (60) that

$$
n \mathfrak{D}=\mathfrak{D} .
$$

Similarly, it can be shown that

$$
(I-n) \mathfrak{D}=\mathfrak{D} .
$$

Additionally, as the distribution $\mathfrak{D}$ is $\bar{\Psi}$-invariant, we have

$$
\bar{\Psi} \mathfrak{D}=\mathfrak{D},
$$

from which

$$
\mathfrak{D}=\bar{\Phi} \mathfrak{D} .
$$

As a consequence of (61), (62) and (63), it is obvious that (54) is correct. Using again the distribution $\mathfrak{D}^{\perp}$ is $\bar{\Psi}$-anti-invariant, we conclude from (9) that (55) is true. Applying (55) in (9), we find

$$
\bar{\Psi} \mathfrak{D}^{\perp}=t \mathfrak{D}^{\perp} .
$$

By virtue of (46) and (47), (56) follows from (64). Therefore, the proof has been shown.

Any semi-invariant submanifold of a golden Riemannian manifold is also characterized by the decomposition of its normal bundle. Thus, we have the following theorem:

Theorem 2. Let $M$ be any submanifold of a golden Riemannian manifold $(\bar{M}, \bar{g}, \bar{\Phi})$. Then $M$ is a semi-invariant submanifold if and only if

$$
T_{p} M^{\perp}=\mathfrak{D}_{p} \oplus \mathfrak{D}_{p}^{\perp}
$$

for each point $p \in M$, where $\mathfrak{D}_{p}$ is the maximal anti-invariant subspace of $T_{p} M^{\perp}$ and $\mathfrak{D}_{p}^{\perp}$ is its orthogonal complement in $T_{p} M^{\perp}$.

Proof. We suppose that $M$ is a semi-invariant submanifold. Then its tangent bundle $T M$ is given by the decomposition $T M=D \oplus D^{\perp}$, where $D$ and $D^{\perp}$ are $\bar{\Phi}$-invariant and $\bar{\Phi}$-anti-invariant distributions, respectively. Let us consider the distribution $\mathfrak{D}^{\perp}=\bar{\Phi} D^{\perp}$ and its orthogonal complementary distribution $\mathfrak{D}$ in $T M^{\perp}$. From Proposition 4, we have two orthogonal complementary distributions $\bar{\Psi}$-invariant $\mathfrak{D}$ and $\bar{\Psi}$-anti-invariant $\mathfrak{D}^{\perp}$ on the submanifold $M$, where $\bar{\Psi}$ is an endomorphism defined by rule $\bar{\Psi}=I-\bar{\Phi}$.

Conversely, we assume that the normal bundle admits $T_{p} M^{\perp}=\mathfrak{D}_{p} \oplus \mathfrak{D}_{p}^{\perp}$ for each point $p \in M$ such that $\mathfrak{D}_{p}$ is the maximal anti-invariant subspace of $T_{p} M^{\perp}$ and $\mathfrak{D}_{p}^{\perp}$ is its orthogonal complement in $T_{p} M^{\perp}$, that is, $\mathfrak{D}$ and $\mathfrak{D}^{\perp}$ are $\bar{\Psi}$-invariant and $\bar{\Psi}$-anti-invariant distributions, respectively. We set $D^{\perp}=\bar{\Psi} \mathfrak{D}^{\perp}$ and denote by $D$ its orthogonal complementary subbundle in $T M$. Let be $X$ in $\Gamma\left(D^{\perp}\right)$. Then there exists a vector field $U \in \Gamma\left(\mathfrak{D}^{\perp}\right)$ such that $X=\bar{\Psi} U$. Thus, we obtain

$$
\bar{\Phi} X=-U \in \Gamma\left(\mathfrak{D}^{\perp}\right) \subseteq \Gamma\left(T M^{\perp}\right),
$$


which shows that $D^{\perp}$ is $\bar{\Phi}$-anti-invariant distribution. Now, we suppose that $X$ belongs to $\Gamma(D)$. Then we get from (3) and (9) that

$$
\bar{g}(\bar{\Phi} X, V)=\bar{g}(X, \bar{\Phi} V)=\bar{g}(X, \operatorname{tr} V)+\bar{g}(X, t \mathfrak{s} V)
$$

for any vector field $V$ in $T M^{\perp}$, where $\mathfrak{r}$ and $\mathfrak{s}$ are the projection operators of the normal bundle $T M^{\perp}$ onto the distributions $\mathfrak{D}$ and $\mathfrak{D}^{\perp}$, respectively. Using (53) and (56) in (65), we obtain

$$
\bar{g}(\bar{\Phi} X, V)=0,
$$

from which it means that

$$
\bar{\Phi} X \in \Gamma(T M) .
$$

Hence, in view of (3), it is easily seen that

$$
\bar{g}(\bar{\Phi} X, Y)=\bar{g}(X, \bar{\Phi} Y)=0
$$

for any vector field $Y \in \Gamma\left(D^{\perp}\right)$. That is, we have

$$
\bar{\Phi} X \in \Gamma(D) .
$$

Thus, (66) and (67) mean that $D$ is a $\bar{\Phi}$-invariant distribution. As a result, the distributions $D$ and $D^{\perp}$ imply that $M$ is a semi-invariant submanifold.

Theorem 3. Let $M$ be any submanifold of a golden Riemannian manifold $(\bar{M}, \bar{g}, \bar{\Phi})$. Then a necessary and sufficient condition for the submanifold $M$ to be semi-invariant is that

$$
t(I-n)=0
$$

Proof. We suppose that $M$ is a semi-invariant submanifold of the golden Riemannian manifold $(\bar{M}, \bar{g}, \bar{\Phi})$. Then its normal bundle has the decomposition $T M^{\perp}=\mathfrak{D} \oplus \mathfrak{D}^{\perp}$, where $\mathfrak{D}$ is $\bar{\Psi}$-invariant distribution and $\mathfrak{D}^{\perp}$ is $\bar{\Psi}$-anti-invariant distribution. We denote by $\mathfrak{r}$ and $\mathfrak{s}$ the projection operators of the normal bundle $T M^{\perp}$ onto the distributions $\mathfrak{D}$ and $\mathfrak{D}^{\perp}$, respectively. Then we have

$$
\mathfrak{r}+\mathfrak{s}=I, \mathfrak{r}^{2}=\mathfrak{r}, \mathfrak{s}^{2}=\mathfrak{s} \text { and } \mathfrak{r} \mathfrak{s}=\mathfrak{s} \mathfrak{r}=0 .
$$

Thus, any vector field $U \in \Gamma\left(T M^{\perp}\right)$ is given by the decomposition

$$
U=\mathfrak{r} U+\mathfrak{s} U
$$

From (69), $\bar{\Psi} U$ can be expressed in the form

$$
\bar{\Psi} U=\bar{\Psi} \mathfrak{r} U+\bar{\Psi} \mathfrak{s} U
$$

for any vector field $U \in \Gamma\left(T M^{\perp}\right)$. Then we get from (9), (53) and (55) that

$$
-t U+(I-n) U=-t \mathfrak{s} U+(I-n) \mathfrak{r} U
$$

for any vector field $U \in \Gamma\left(T M^{\perp}\right)$. Hence, identifying the tangential and normal parts in (70), respectively, it is shown that

$$
t=t \mathfrak{s} U \text { and }(I-n)=(I-n) \mathfrak{r} .
$$


Thus, we obtain from (14) and (53) that

$$
t(I-n)=t(I-n) \mathfrak{r}=T t \mathfrak{r}=0 .
$$

Conversely, we assume that $M$ is a submanifold of the golden Riemannian manifold $(\bar{M}, \bar{g}, \bar{\Phi})$ and $t(I-n)=0$. Applying the endomorphism $(I-n)$ from the right hand side to (15), we get

$$
n^{3}=2 n^{2}-I
$$

Let us consider two operators $\mathfrak{r}$ and $\mathfrak{s}$ defined by

$$
\mathfrak{r}=n^{2}-n
$$

and

$$
\mathfrak{s}=-n^{2}+n+I,
$$

respectively. Then the operators $\mathfrak{r}$ and $\mathfrak{s}$ verify that

$$
\mathfrak{r}+\mathfrak{s}=I, \mathfrak{r}^{2}=\mathfrak{r}, \mathfrak{s}^{2}=\mathfrak{s} \text { and } \mathfrak{r} \mathfrak{s}=\mathfrak{s} \mathfrak{r}=0,
$$

which demonstrate that $\mathfrak{r}$ and $\mathfrak{s}$ are orthogonal complementary projection operators. Hence, there are two orthogonal complementary distributions $\mathfrak{D}$ and $\mathfrak{D}^{\perp}$ corresponding to the projection operators $\mathfrak{r}$ and $\mathfrak{s}$, respectively. Under the assumption that $t(I-n)=0$, we derive from (71), (72) and (73) that

$$
(I-n) \mathfrak{r}=(I-n),(I-n) \mathfrak{s}=0, \mathfrak{s}(I-n) \mathfrak{r}=\mathfrak{s}(I-n)=0 \text { and } \mathfrak{t}=0,
$$

which imply that the distribution $\mathfrak{D}$ is $\bar{\Psi}$-invariant and the distribution $\mathfrak{D}^{\perp}$ is $\bar{\Psi}$-anti-invariant. Thus, we have two orthogonal complementary distributions $\bar{\Psi}$-invariant $\mathfrak{D}$ and $\bar{\Psi}$-anti-invariant $\mathfrak{D}^{\perp}$ on the submanifold $M$. In other words, $M$ is a semi-invariant submanifold.

Proposition 6. Let $M$ be any semi-invariant submanifold of a golden Riemannian manifold $(\bar{M}, \bar{g}, \bar{\Phi})$. Then the following relations are verified:

$$
\operatorname{ker} t=\operatorname{ker}\left(n^{2}-n-I\right)=\operatorname{ker}(N t)
$$

and

$$
\operatorname{ker}(I-n)=\operatorname{ker}\left(n-n^{2}\right)=\operatorname{ker}(N t-I) .
$$

Proof. The proof can be shown in a manner similar to that of Proposition 2.

Proposition 7. Let $M$ be any semi-invariant submanifold of a golden Riemannian manifold $(\bar{M}, \bar{g}, \bar{\Phi})$. Then the following relations are satisfied:

$$
\mathfrak{D}=\operatorname{ker} t=\operatorname{ker}\left(n^{2}-n-I\right)=\operatorname{ker}(N t)
$$

and

$$
\mathfrak{D}^{\perp}=\operatorname{ker}(I-n)=\operatorname{ker}\left(n-n^{2}\right)=\operatorname{ker}(N t-I) .
$$

Proof. Taking account of Propositions 5 and 6, the proof is easily obtained.

Now, we give an example to illustrate our results. 
Example 1. We consider a tensor field $\bar{\Phi}$ of type $(1,1)$ on 4 -dimensional Euclidean space $\left(\mathbb{R}^{4},\langle\rangle,\right)$ with local coordinates $\left(x_{1}, x_{2}, x_{3}, x_{4}\right)$ defined by

$$
\bar{\Phi}\left(\frac{\partial}{\partial x_{1}}, \frac{\partial}{\partial x_{2}}, \frac{\partial}{\partial x_{3}}, \frac{\partial}{\partial x_{4}}\right)=\left(\phi \frac{\partial}{\partial x_{1}}, \phi \frac{\partial}{\partial x_{2}},(1-\phi) \frac{\partial}{\partial x_{3}},(1-\phi) \frac{\partial}{\partial x_{4}}\right),
$$

where $\phi$ and $1-\phi$ are the roots of the algebraic equation $x^{2}=x+1$. Then it is obvious that $\left(\mathbb{R}^{4},\langle\rangle,, \bar{\Phi}\right)$ is a golden Riemannian manifold. Let $M$ be a submanifold in the ambient manifold $\left(\mathbb{R}^{4},\langle\rangle,, \bar{\Phi}\right)$ given by

$$
M=\left\{(u, v, \phi \cos v, \phi \sin v): u, v \in\left(0, \frac{\pi}{2}\right)\right\} .
$$

By a direct calculation, it can be obtained that

$$
T M=\left\{E_{1}=\frac{\partial}{\partial x_{1}}, E_{2}=\frac{\partial}{\partial x_{2}}-\phi \sin v \frac{\partial}{\partial x_{3}}+\phi \cos v \frac{\partial}{\partial x_{4}}\right\}
$$

and

$$
T M^{\perp}=\left\{N_{1}=\phi \frac{\partial}{\partial x_{2}}+\sin v \frac{\partial}{\partial x_{3}}-\cos v \frac{\partial}{\partial x_{4}}, N_{2}=-\cos v \frac{\partial}{\partial x_{3}}+\sin v \frac{\partial}{\partial x_{4}}\right\} .
$$

If we choose the distributions $D$ and $D^{\perp}$ such that $D=\operatorname{Span}\left\{E_{1}\right\}$ and $D^{\perp}=\operatorname{Span}\left\{E_{2}\right\}$, then the tangent bundle $T M$ has the decomposition $T M=D \oplus D^{\perp}$. On the other hand, it seems that

$$
\bar{\Phi}\left(E_{1}\right)=\phi E_{1} \in \Gamma(D)
$$

and

$$
\bar{\Phi}\left(E_{2}\right)=N_{1} \in \Gamma\left(T M^{\perp}\right),
$$

from which $D$ and $D^{\perp}$ are $\bar{\Phi}$-invariant and $\bar{\Phi}$-anti-invariant distributions, respectively. Hence, $M$ is a semi-invariant submanifold. It can be also shown that $\mathfrak{D}=\operatorname{Span}\left\{N_{2}\right\}$ and $\mathfrak{D}^{\perp}=\operatorname{Span}\left\{N_{1}\right\}$ such that $T M^{\perp}=\mathfrak{D} \oplus \mathfrak{D}^{\perp}$. Furthermore, for any vector fields $X \in \Gamma(T M)$ and $U \in \Gamma\left(T M^{\perp}\right)$, we get

$$
\bar{\Phi} X=\alpha \phi E_{1}+\beta N_{1}, \alpha, \beta \in \mathbb{R}
$$

and

$$
\bar{\Phi} U=\lambda\left(E_{2}+N_{1}\right)+\mu(1-\phi) N_{2}, \lambda, \mu \in \mathbb{R} .
$$

Thus, the operators $T, N, t$ and $n$ are as follows:

$$
\begin{gathered}
T X=\alpha \phi E_{1}, \\
N X=\beta N_{1} \\
t U=\lambda E_{2}
\end{gathered}
$$

and

$$
n U=\lambda N_{1}+\mu(1-\phi) N_{2}
$$

for any vector fields $X \in \Gamma(T M)$ and $U \in \Gamma\left(T M^{\perp}\right)$, respectively. Consequently, it is easy to check that the expressions of Proposition 1, Theorem 1, Proposition 2, Proposition 3, Proposition 4, Proposition 5, Theorem 2, Theorem 3, Proposition 6 and Proposition 7 hold.

Author Contributions: Conceptualization, M.G. and E.K.; Investigation, M.G.; Methodology, M.G., E.K. and S.K.; Supervision, M.G. and E.K.; Visualization, M.G.; Writing-original draft preparation, M.G.; Writing-review and editing, M.G., E.K. and S.K. 
Funding: This research received no external funding.

Conflicts of Interest: The authors declare no conflict of interest.

\section{References}

1. Bejancu, A. CR Submanifolds of a Kaehler Manifold. I. Proc. Am. Math. Soc. 1978, 69, 135-142. [CrossRef]

2. Bejancu, A.; Papaghiuc, N. Semi-Invariant Submanifolds of a Sasakian Manifold. An. Ştint. Univ. Al. I. Cuza Iaşi. Mat. (N.S.) 1981, 27, 163-170.

3. Papaghiuc, N. Semi-Invariant Submanifolds in a Kenmotsu Manifold. Rend. Mat. 1983, 3, 607-622.

4. Bejancu, A. Semi-Invariant Submanifolds of Locally Riemannian Product Manifolds. Ann. Univ. Timisoara S. Math. 1984, 22, 3-11.

5. Bejancu, A.; Papaghiuc, N. Semi-Invariant Submanifolds of a Sasakian Space Form. Colloq. Math. 1984, 48, 77-88. [CrossRef]

6. Bejan, C.L. Almost Semi-Invariant Submanifolds of a Cosymplectic Manifold. An. Ştint. Univ. Al. I. Cuza Iaşi. Mat. (N.S.) 1985, 31, 149-156.

7. Cabras, A.; Matzeu, P. Almost Semi-Invariant Submanifolds of a Cosymplectic Manifold. Demonstratio Math. 1986, 19, 395-401. [CrossRef]

8. Kobayashi, M. Semi-Invariant Submanifolds of a Certain Class of Almost Contact Manifolds. Tensor (N.S.) 1986, 43, 28-36.

9. Matsumoto, K.; Shahid, M.H.; Mihai, I. Semi-Invariant Submanifolds of Certain Almost Contact Manifolds. Bull. Yamagata Univ. Nat. Sci. 1994, 13, 183-192.

10. Shahid, M.H. On Semi-Invariant Submanifolds of a Nearly Sasakian Manifold. Indian J. Pure Appl. Math. 1993, 24, 571-580.

11. Prasad, B. Semi-Invariant Submanifolds of a Lorentzian Para-Sasakian Manifold. Bull. Malaysian Math. Soc. 1998, 21, 21-26.

12. Kim, S.J.; Lin, X.; Tripathi, M.M. On Semi-Invariant Submanifolds of Nearly Trans-Sasakian Manifolds. Int. J. Pure Appl. Math. 2004, 1, 15-34.

13. Alegre, P. Semi-Invariant Submanifolds of Lorentzian Sasakian Manifolds. Demonstratio Math. 2011, 44, 391-406. [CrossRef]

14. Crâşmăreanu, M.C.; Hreţcanu, C.E. Golden Differential Geometry. Chaos Soliton. Fract. 2008, 38, $1229-1238$. [CrossRef]

15. Hreţcanu, C.E.; Crâşmăreanu, M.C. On Some Invariant Submanifolds in a Riemannian Manifold with Golden Structure. An. Ştiinţ. Univ. Al. I. Cuza Iaşi. Mat. (N.S.) 2007, 53, 199-211.

16. Hreţcanu, C.E.; Crâşmăreanu, M.C. Applications of the Golden Ratio on Riemannian Manifolds. Turk. J. Math. 2009, 33, 179-191.

17. Blaga, A.M.; Hreţcanu, C.E. Invariant, Anti-Invariant and Slant Submanifolds of a Metallic Riemannian Manifold. Novi Sad J. Math. 2018, 48, 55-80. [CrossRef]

18. Hreţcanu, C.E.; Blaga, A.M. Slant and Semi-Slant Submanifolds in Metallic Riemannian Manifolds. J. Funct. Spaces 2018, 2018, 2864263. [CrossRef]

19. Hrețcanu, C.E.; Blaga, A.M. Hemi-Slant Submanifolds in Metallic Riemannian Manifolds. Carpathian J. Math. 2019, 35, 59-68.

20. Erdoğan, F.E.; Yıldırım, C. On a Study of the Totally Umbilical Semi-Invariant Submanifolds of Golden Riemannian Manifolds. J. Polytechnic 2018, 21, 967-970. [CrossRef]

21. Goldberg, S.I.; Yano, K. Polynomial Structures on Manifolds. Kodai Math. Sem. Rep. 1970, 22, $199-218$. [CrossRef] 